\title{
FIVE-LOOP CRITICAL EXPONENTS OF THE WEAKLY DILUTED ISING MODEL: 3D APPROACH VERSUS $\sqrt{\varepsilon}$-EXPANSION
}

\author{
R. Folk ${ }^{1}$, Yu. Holovatch ${ }^{2}$, T. Yavors'kii ${ }^{3}$ \\ ${ }^{1}$ Institut für Theoretische Physik Johannes Kepler Universität Linz A-4040, Linz, Austria \\ ${ }^{2}$ Institute for Condensed Matter Physics of the Ukrainian Academy of Sciences \\ 1 Svientsitskii Str., UA-290011 Lviv, Ukraine \\ ${ }^{3}$ Ivan Franko Lviv State University, Chair for Theoretical Physics \\ 12 Drahomanov Str., UA-290005 Lviv, Ukraine
}

(Received April 9, 1998)

\begin{abstract}
Based on the recently obtained five-loop renormalization group functions of the $\phi^{4}$-theory with $O(n)$-symmetric and cubic interactions, we analyse these functions for the weakly diluted Ising model case $n=0$ both by means of the $\sqrt{\varepsilon}$-expansion and in the fixed $d=3$ technique. We apply different resummation procedures and report numerical values for the critical exponents dependent on the order of approximation. We compare the convergence of the results obtained in the framework of the two schemes.
\end{abstract}

Key words: Ising model, quenched disorder, renormalization group, asymptotic series, critical exponents.

PACS number(s): 64.60.Ak, 61.43.-j, 11.10.Gh

\section{INTRODUCTION}

The study of the critical behaviour of the threedimensional Ising model (3DIM) with weak quenched disorder [1] is of great interest. This simplest spin model with the universal properties sensible to the weak dilution [2], allows to investigate the principle peculiarities of the disorder effect without shading the matter of problem by details. There are experimental [3-5] values of the critical exponents as well as results of Monte Carlo [6-10] and renormalization group Monte Carlo simulations [11] which prove the appearance of a new set of critical exponents for this model. Their numerical estimates restrict the values so that one can test various theoretical approaches for calculating these values within renormalization group (RG) theory.

The progress in the study of the critical phenomena in disordered spin systems was outlined by applying the RG approach which reduced the task of calculating the critical exponents to the investigation of the critical properties of a field theoretical Landau-Ginzburg model Hamiltonian with two $\phi^{4}$-terms of different symmetry $[12,14]$ However, in the case of the diluted Ising model the first attempts to perform a $\sqrt{\varepsilon}$-expansion appeared to be ineffective for obtaining reasonable numerical values of the critical exponents of the model [12-16].

Meanwhile the field-theoretical RG approach was successfully applied to the Landau-Ginzburg model Hamiltonian with one coupling constant [17-23] describing the critical behaviour of the pure model. The asymptotic nature of the RG-functions expansions in coupling as well as of the $\varepsilon$-expansion was proven [24-26]. Thus new methods of treating the calculated field theoretic functions were required. The proof of the Borel-summability of expansions [27] allowed to use either simple resummation techniques of Padé-Borel type which do not need the knowledge of asymptotic behaviour of the higher order terms (see e.g. $[28,29]$ ), or more complex techniques which need such an information [30,31]. From both types of resummation techniques accurate values of the pure 3 DIM critical exponents $[22,23,29,31]$ were obtained.

In the case of the diluted Ising model the applicability of resummation techniques still remains an open question, since the nature of the expansion of the RG-functions in two couplings and of the $\sqrt{\varepsilon}$-expansion so far is unknown. However, the direct application of the resummation to $\mathrm{RG}$-functions with two variables in the case of the massive fixed-dimension RG scheme [33-38] as well as in the minimal subtraction scheme at $d=3$ [39] appeared to be strongly effective.

Our study has been motivated by recent results [40] for the RG-functions of the $\phi^{4}$-theory with $O(n)$-symmetric and cubic interactions which enables one to extract the RG-functions for the weakly diluted Ising model in five loop order. These functions were obtained within the minimal subtraction scheme and can be analysed by means of the $\sqrt{\varepsilon}$-expansion [41]. Within the fixed $d$ technique a procedure for the model with one coupling was proposed in Ref. [42,43] and is applicable for the dilute case too. This possibility permits one to compare the convergence properties of the results obtained by different methods.

In this paper, we will report briefly the numerical values of critical exponents for different orders of approximation and estimate the convergence of the results. We will proceed in two steps: first we sum up the threedimensional RG-functions and then we sum up directly the $\sqrt{\varepsilon}$-expansion. Comparing the results with the values of the corresponding analysis of the $\phi^{4}$-theory with one coupling we estimate the reliability and effectiveness of the resummation schemes for the dilute model in the two cases. 


\section{THE MODEL AND THE RG-PROCEDURE}

As it is well known, the critical behaviour of the quenched weakly-diluted Ising model in the Euclidian space of $d=4-\varepsilon$ dimensions is governed by a Hamiltonian with two coupling constants [14]:

$$
\begin{aligned}
\mathcal{H}(\phi) & =\int \mathrm{d}^{d} R\left\{\frac{1}{2} \sum_{\alpha=1}^{n}\left[\left|\nabla \phi_{\alpha}\right|^{2}+m_{0}^{2} \phi_{\alpha}^{2}\right]\right. \\
& \left.-\frac{v_{0}}{4 !}\left(\sum_{\alpha=1}^{n} \phi_{\alpha}^{2}\right)^{2}+\frac{u_{0}}{4 !} \sum_{\alpha=1}^{n} \phi_{\alpha}^{4}\right\},
\end{aligned}
$$

in replica limit $n \rightarrow 0$. Here $\phi$ is a scalar order parameter; $u_{0}>0, v_{0}>0$ are bare coupling constants; $m_{0}$ is bare mass.

In order to extract critical properties of a model described by (1), one passes from the bare parameters $m_{0}, u_{0}$ and $v_{0}$ to the corresponding renormalized quantities $m, u, v$, which in the framework of the minimal subtraction scheme are defined via relations:

$$
\begin{aligned}
& m_{0}^{2}=\frac{Z_{2}}{Z_{\phi}} m^{2}, \\
& u_{0}=\mu^{\epsilon} \frac{Z_{4, u}}{Z_{\phi}^{2}} u, \\
& v_{0}=\mu^{\epsilon} \frac{Z_{4, v}}{Z_{\phi}^{2}} v,
\end{aligned}
$$

where $\mu$ is the external momentum scale and $Z_{\phi}, Z_{2}, Z_{4, u}, Z_{4, v}$ are the renormalization constants.

Introducing the RG-functions by the following definitions:

$$
\begin{aligned}
& \beta_{u}(u, v)=\left.\mu \frac{\partial u}{\partial \mu}\right|_{m_{0}, u_{0}, v_{0}}, \\
& \beta_{v}(u, v)=\left.\mu \frac{\partial v}{\partial \mu}\right|_{m_{0}, u_{0}, v_{0}},
\end{aligned}
$$

$$
\begin{aligned}
\gamma_{2}(u, v) & =\left.\mu \frac{\partial \ln Z_{\phi}^{1 / 2}}{\partial \mu}\right|_{m_{0}, u_{0}, v_{0}}, \\
\gamma_{m}(u, v) & =\left.\mu \frac{\partial \ln m}{\partial \mu}\right|_{m_{0}, u_{0}, v_{0}},
\end{aligned}
$$

the critical exponents $\eta$ and $\nu$ of the pair correlation function at $T_{c}$ and of the correlation length respectively are given by

$$
\begin{aligned}
\eta & =2 \gamma_{2}\left(u^{*}, v^{*}\right), \\
1 / \nu & =2\left(1-\gamma_{m}\left(u^{*}, v^{*}\right)\right),
\end{aligned}
$$

where $\left\{u^{*}, v^{*}\right\}$ denotes the stable fixed point. This fixed point is the solution of the set of equations:

$$
\begin{aligned}
& \beta_{u}\left(u^{*}, v^{*}\right)=0, \\
& \beta_{v}\left(u^{*}, v^{*}\right)=0,
\end{aligned}
$$

with positive real parts of the eigenvalues of the stability matrix $B=\partial \beta_{u_{i}} /\left.\partial u_{j}\right|_{u_{i}^{*}}, u_{i} \equiv\{u, v\}$.

However, for the sake of convenience one often deals with the expansions of some combinations of the critical exponents instead of working with them directly (3). The values listed below have been calculated by resumming the value $1 / \nu-1=1-2 \gamma_{m}$ and the inverse value of the magnetic susceptibility critical exponents $\gamma^{-1}=\left(1-\gamma_{m}\right) /\left(1-\gamma_{2}\right)$; the numerical values of the rest of the critical exponents were obtained by familiar scaling laws.

\section{THE RESUMMATION AND THE RESULTS}

In this chapter we obtain the critical exponents of the weakly diluted quenched Ising model by the methods mentioned above. The RG-functions of the corresponding Hamiltonian (1) can be obtained in the five-loop approximation from the appropriate expressions for $\mathrm{RG}^{-}$ functions of the $\phi^{4}$-theory with $O(n)$-symmetric and $\mathrm{cu}^{-}$ bic interactions [40]. In the replica limit $n=0$ the results of Ref. [40] read:

$$
\begin{aligned}
\beta_{u} / u & =-\varepsilon+3 u-4 v-17 / 3 u^{2}+46 / 3 v u-82 / 9 v^{2} \\
& +32.54968284 u^{3}-123.1987313 v u^{2}+158.1816418 v^{2} u-60.32526811 v^{3} \\
& -271.6057842 u^{4}+1318.116311 v u^{3}-2452.429994 v^{2} u^{2}+2003.560971 v^{3} u \\
& -559.7143854 v^{4}+2848.568254 u^{5}-16789.89843 v u^{4}+40367.08593 v^{2} u^{3} \\
& -48971.12730 v^{3} u^{2}+29091.77179 v^{4} u-6377.751189 v^{5}, \\
\beta_{v} / v & =-\varepsilon-8 / 3 v+2 u-14 / 3 v^{2}+22 / 3 v u-5 / 3 u^{2}
\end{aligned}
$$




$$
\begin{aligned}
& -25.45714897 v^{3}+62.25499170 v^{2} u-36.36645522 v u^{2}+7 u^{3} \\
& -200.9263690 v^{4}+667.3761895 v^{3} u-650.5641816 v^{2} u^{2}+259.2586891 v u^{3} \\
& -39.91261012 u^{4}-2003.976188 v^{5}+8469.158907 v^{4} u-11721.60876 v^{3} u^{2} \\
& +7434.635066 v^{2} u^{3}-2344.277996 v u^{4}+301.5110976 u^{5} \\
\gamma_{2} & =1 / 18 v^{2}-1 / 6 v u+1 / 12 u^{2}+1 / 27 v^{3}-1 / 6 v^{2} u+3 / 16 v u^{2}-1 / 16 u^{3} \\
& +125 / 648 v^{4}-125 / 108 v^{3} u+145 / 72 v^{2} u^{2}-65 / 48 v u^{3}+65 / 192 u^{4} \\
& +1.005978154 v^{5}-7.544836154 v^{4} u+18.04854621 v^{3} u^{2} \\
& -19.07838990 v^{2} u^{3}+9.627924878 v u^{4}-1.925584976 u^{5} \\
\gamma_{m} & =-1 / 3 v+1 / 2 u-5 / 18 v^{2}+5 / 6 v u-5 / 12 u^{2}-37 / 36 v^{3}+37 / 8 v^{2} u \\
& -251 / 48 v u^{2}+7 / 4 u^{3}-5.3808017 v^{4}+32.28481 v^{3} u-57.177011 v^{2} u^{2} \\
& +39.765731 v u^{3}-9.97815253 u^{4}-37.850485 v^{5} \\
& +283.878638 v^{4} u-686.375317 v^{3} u^{2}+737.493196 v^{2} u^{3} \\
& -376.1776339 v u^{4}+75.37777445 u^{5} .
\end{aligned}
$$

The $\beta$-functions (5)-(6) are degenerated at the one-loop level resulting in an expansion in $\sqrt{\varepsilon}[12-16]$ in the case of the diluted Ising model. The expansion was obtained in five-loop order [41] and for the critical exponents it reads:

$$
\begin{aligned}
& \nu=1 / 2+0.08411582 \varepsilon^{1 / 2}-0.01663203 \varepsilon+0.04775351 \varepsilon^{3 / 2}+0.27258431 \varepsilon^{2} \\
& \eta=-0.00943396 \varepsilon+0.03494350 \varepsilon^{3 / 2}-0.04486498 \varepsilon^{2}+0.02157321 \varepsilon^{5 / 2} \\
& \gamma=1+0.16823164 \varepsilon^{1 / 2}-0.02854708 \varepsilon+0.07882881 \varepsilon^{3 / 2}+0.56450490 \varepsilon^{2}
\end{aligned}
$$

As it was stated in the introduction, in order to calculate the critical exponents from these expressions we have to adopt the resummation procedures, in particular those which do not need the knowledge of the high order behaviour of the expansions terms. The restriction is motivated by the fact that such an information is still unavailable. However, we start our study from the well-established case of the pure Ising system (scalar $\phi^{4}$ model) where the asymptotic nature of the RG-functions and $\varepsilon$-expansion is proven [27] and the large-order behaviour has been calculated [24-26].

The analysis is introduced by representing the expressions for the critical exponents $\nu$ and $\gamma$ of the pure Ising model in the form of the Padé-approximant: $[M / N](x)=\sum_{i=0}^{M} a_{i} x^{i} / \sum_{j=0}^{N} b_{j} x^{j}$. The exponents in the five-loop approximation where obtained in Ref. [23], they read:

$$
\begin{aligned}
\nu & =1 / 2+1 / 12 \varepsilon+0.04320988 \varepsilon^{2}-0.01904337 \varepsilon^{3} \\
& +0.07088376 \varepsilon^{4}-0.21701787 \varepsilon^{5},
\end{aligned}
$$

$$
\begin{aligned}
\gamma & =1+1 / 6 \varepsilon+0.07716049 \varepsilon^{2}-0.04897495 \varepsilon^{3} \\
& +0.14357422 \varepsilon^{4}-0.44662483 \varepsilon^{5}
\end{aligned}
$$

The Padé table for the expressions (10) is given in the table I. The number of the row, $N$, and of the column, $M$, corresponds to the order of the numerator and denominator of the corresponding Padé-approximant $[M / N]$ respectively. In table $I$ as well as in the subsequent tables "o" means that a corresponding Padé-approximant cannot be constructed. One can see from the table the expected convergence of the values in the diagonal and first off-diagonal. We therefrom estimate the values of the critical exponents to be $\nu=0.628, \gamma=1.236$. These values can be compared with the most accurate values $\nu=0.628 \pm 0.001, \gamma=1.234 \pm 0.002$ [22], obtained by means of more sophisticated resummation procedures [30] on the basis of the five-loop $\varepsilon$-expansion. We conclude that this good agreement justifies the application of the Padé-analysis. 


\begin{tabular}{||c|cccccc||}
\hline \hline$\nu$ & 0 & 1 & 2 & 3 & 4 \\
\hline 0 & 0.500 & 0.571 & 0.609 & 0.608 & 0.648 & 0.620 \\
1 & 0.599 & 0.673 & 0.608 & 0.610 & 0 & 0 \\
2 & 0.646 & 0.621 & 0.633 & 0.625 & 0 & 0 \\
3 & 0.597 & 0.631 & 0.627 & 0 & 0 & 0 \\
4 & 0.732 & 0.625 & 0 & 0 & 0 \\
5 & 0.431 & 0 & 0 & 0 & 0 \\
\hline \hline$\gamma$ & 0 & 1 & 2 & 3 & 1.339 \\
1 & 1.2000 & 1.167 & 1.244 & 1.195 & 0 \\
2 & 1.276 & 1.2310 & 1.213 & 1.231 & 0 \\
3 & 1.171 & 1.242 & 1.243 & 0 & 0 \\
4 & 1.440 & 1.227 & 0 & 0 & 0 \\
5 & 0.845 & 0 & 0 & 0 & 0 \\
\hline \hline
\end{tabular}

Table I. The Padé table for the values of the correlation length $(\nu)$ and the susceptibility $(\gamma)$ critical exponents from the $\varepsilon$-expansion of the pure 3DIM.

\begin{tabular}{||c|cccccc||}
\hline \hline$\nu$ & 0 & 1 & 2 & 3 & 4 \\
\hline 0 & 0.500 & 0.560 & 0.584 & 0.592 & 0.599 & 0.602 \\
1 & 0.599 & $0.695 \mathrm{c}$ & 0.604 & - & 0 & 0.601 \\
2 & 0.645 & 0.623 & $0.631 \mathrm{c}$ & 0.628 & 0 & 0 \\
3 & 0.597 & 0.629 & 0.629 & 0 & 0 & 0 \\
4 & 0.732 & 0.629 & 0 & 0 & 0 \\
5 & 0.431 & 0 & 0 & 3 & 1.232 & 0 \\
\hline \hline$\gamma$ & 0 & 1.147 & 1.205 & 1.221 & 0 \\
1 & 1.000 & $1.357 \mathrm{c}$ & 1.208 & 0 & 0 \\
2 & 1.200 & 1.233 & 1.238 & 0 & 0 \\
3 & 1.171 & 1.238 & 1.236 & 0 & 0 \\
4 & 1.440 & 1.234 & 0 & 0 & 0 \\
5 & 0.845 & 0 & 0 & 0 & 0 \\
\hline \hline
\end{tabular}

Table II. The results of the Padé-Borel resummation of the correlation length $(\nu)$ and the susceptibility $(\gamma)$ critical exponents from the $\varepsilon$-expansion of the pure 3DIM. The " $c$ "-subscript denotes that the real part of the corresponding value is given.

\begin{tabular}{|c|c|c|c|c|c|c|}
\hline Loop $(L)$ & $u^{*}$ & $\gamma$ & $\nu$ & $\alpha$ & $\eta$ & $b$ \\
\hline 2 & 0.7061 & 1.2857 & 0.654 & 0.0383 & 0.0339 & 0.5155 \\
\hline 3 & 0.4564 & 1.2264 & 0.621 & 0.1383 & 0.0237 & 0.8696 \\
\hline 4 & 0.5163 & 1.2495 & 0.638 & 0.0846 & 0.0430 & 0.6875 \\
\hline 5 & 0.4561 & 1.2275 & 0.624 & 0.1293 & 0.0315 & 0.9452 \\
\hline
\end{tabular}

Table III. The results of the application of the Padé-Borel resummation ([L/1]) to the RG-functions of the pure 3 DIM. 


\begin{tabular}{||c|ccccc}
\hline \hline$\nu$ & 0 & 1 & 2 & 3 \\
\hline 0 & 0.500 & 0.572 & 0.570 & 0.601 \\
1 & 0.601 & 0.570 & 0.572 & 0.564 \\
2 & 0.560 & 0.586 & 0.565 & 0 & 0.727 \\
3 & 0.640 & 0.541 & 0 & 0 & 0 \\
4 & 1.828 & 0 & 0 & 0 \\
\hline \hline$\gamma$ & 0 & 1 & 1.140 & 3 \\
\hline 0 & 1. & 1.168 & 1.1606 & 0 \\
1 & 1.2023 & 1.1438 & 1.137 & 1.219 & 0 \\
2 & 1.125 & 1.172 & 0 & 0 & 0 \\
3 & 1.257 & 1.101 & 0 & 0 & 0 \\
4 & 3.824 & 0 & 0 & 0 \\
\hline \hline
\end{tabular}

Table IV. The Padé table for the values of the correlation length $(\nu)$ and the susceptibility $(\gamma)$ critical exponents from the $\sqrt{\varepsilon}$-expansion of the 3DIM with quenched weak disorder.

\begin{tabular}{|c|c|c|c|c|c|}
\hline$\nu$ & 0 & 1 & 2 & 3 & 4 \\
\hline 0 & 0.500 & 0.563 & 0.569 & 0.577 & 0.592 \\
\hline 1 & 0.601 & 0.573 & $0.559 \mathrm{c}$ & - & o \\
\hline 2 & 0.560 & 0.584 & $0.568 \mathrm{C}$ & o & o \\
\hline 3 & 0.639 & $0.529 \mathrm{c}$ & o & o & o \\
\hline 4 & 1.828 & o & $\mathrm{o}$ & o & $\mathrm{O}$ \\
\hline$\overline{\gamma \gamma}$ & 0 & 1 & 2 & 3 & 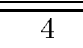 \\
\hline 0 & 1.000 & 1.149 & 1.148 & 1.176 & 1.252 \\
\hline 1 & 1.202 & 1.148 & 1.149 & 1.000 & o \\
\hline 2 & 1.125 & 1.168 & $1.141 \mathrm{c}$ & o & o \\
\hline 3 & 1.257 & $1.086 \mathrm{c}$ & o & o & o \\
\hline 4 & 3.824 & o & o & o & o \\
\hline
\end{tabular}

Table V. The results of the Padé-Borel resummation of the correlation length $(\nu)$ and the susceptibility $(\gamma)$ critical exponents from the $\sqrt{\varepsilon}$-expansion of the 3DIM with quenched weak disorder. The " $c$ "-subscript denotes that the real part of the corresponding value is given.

\begin{tabular}{||c|cccccccc}
\hline \hline Loop & $u^{*}$ & $v^{*}$ & $\gamma$ & $\nu$ & $\alpha$ & $\eta$ & $b_{1}$ \\
\hline 2 & 0.7962 & 0.0862 & 1.312 & 0.668 & -0.0032 & 0.0346 & 0.0985 & 0.5100 \\
3 & 0.6957 & 0.2544 & 1.291 & 0.652 & 0.0432 & 0.0209 & 0.4599 & 0.9933 \\
4 & 0.7046 & 0.1360 & 1.319 & 0.677 & -0.0321 & 0.0526 & $0.330 \mathrm{c}$ & $0.330 \mathrm{c}$ \\
5 & - & - & - & - & - & - & - & - \\
\hline \hline
\end{tabular}

Table VI. The results of the application of the Chisholm-Borel resummation ([L/1]) to the RG-functions of the 3DIM with quenched weak disorder.

As another procedure we apply the Padé-Borel resummation technique to the $\varepsilon$-expansion (10) of the pure 3 DIM critical exponents. The procedure consists in several steps: 
- starting from the initial sum $S$ of $n$ terms one constructs its Borel-Leroy image:

$$
S=\sum_{i=0}^{n} a_{i} x^{i} \Rightarrow \sum_{i=0}^{n} \frac{a_{i}(x t)^{i}}{\Gamma(i+p+1)}
$$

where $\Gamma(x)$ is the Euler's gamma function and $p$ is an arbitrary non-negative number [44];

- the Borel-Leroy image (11) is extrapolated by a rational Padé approximant $[M / N](x t)$;

- the resummed function $S^{\text {res }}$ is obtained in the form:

$$
S^{r e s}=\int_{0}^{\infty} d t \exp (-t) t^{p}[M / N](x t)
$$

The values of the critical exponents $\nu$ and $\gamma$ obtained with the Padé-Borel resummation applying the general approximant are given in table II. Note however that the most stable results are obtained for the approximants with a linear denominator (second columns), which reconstitutes the sign-alternating behaviour of the general terms of the initial expressions [25]. The estimates for the exponents $\nu=0.629, \gamma=1.236$ are in good agreement either with the above results of Padé-analysis as well as with the data of [22].

The third method we apply consists in repeating the same analysis as above to the $\mathrm{RG}$-functions calculated directly at $d=3$. We use here the Padé-Borel resummation technique with a linear denominator approximant [45]. The corresponding values are shown in table III [46]. One may compare them with the results obtained by the analysis of the six-loop RG-functions in the $d=3$ massive field-theoretical approach, $\nu=0.630 \pm 0.0020, \gamma=$ $1.241 \pm 0.0040[29], \nu=0.630 \pm 0.0015, \gamma=1.241 \pm 0.0020$ [31] and the results of $d=3$ minimal subtraction scheme in the five-loop approximation $\nu=0.629 \pm 0.005, \gamma=$ $1.235 \pm 0.005[43]$.

Having verified the validity of the Padé and the PadéBorel resummation techniques in the simpler case of the pure Ising model, we now turn to the results of their applications to the $\sqrt{\varepsilon}$-expansions (9) and the $3 d$ theory RG-functions (5)-(8) of the weakly diluted Ising model. The appropriate values for the $\sqrt{\varepsilon}$-expansions $(9)$ are listed in the tables IV-V in the same notations as in the tables (I-II) [47]. One can see from the tables that both the experimental $(\nu=0.70 \pm 0.02, \gamma=1.37 \pm 0.04$ [5]) and reliable theoretical $(\nu=0.67, \gamma=1.326$ [35]; $\nu=0.6714, \gamma=1.321$ [36]) values are not obtained. Comparing these results with the corresponding data for the pure Ising model we conclude that the nature of the $\sqrt{\varepsilon}$-expansion series does not allow to obtain reliable information at $d=3$ by means of the methods mentioned for the case of pure Ising model. This can be considered as indirect evidence of the non-asymptotic nature of the $\sqrt{\varepsilon}$-expansion. Thus a different kind of analysis for the $\sqrt{\varepsilon}$-expansion has to be developed.
In order to treat the theory directly at $d=3$ we apply the so called Chisholm-Borel resummation technique to the RG-functions of the weakly diluted Ising model, which depend on two variables. The Chisholm-Borel resummation procedure can be defined as follows:

- constructing the Borel-Leroy image of the initial sum $S$ of two variables $u$ and $v$ :

$$
S=\sum_{0 \leq i+j \leq n} a_{i, j} u^{i} v^{j} \Rightarrow \sum_{0 \leq i+j \leq n} \frac{a_{i, j}(u t)^{i}(v t)^{j}}{\Gamma(i+j+p+1)}
$$

where $\Gamma(x)$ is the Euler's gamma function and $p$ is an arbitrary non-negative number [44];

- extrapolating the Borel-Leroy image (13) by a rational Chisholm approximant $[M / N](u t, v t)$ which can be defined as a ratio of two polynomials both in variables $u$ and $v$, of degree $M$ and $N$ so that the first terms of its expansion are equal to those of the function which is approximated;

- the resummed function $S^{\text {res }}$ obtain the form:

$$
S^{r e s}=\int_{0}^{\infty} d t \exp (-t) t^{p}[M / N](u t, v t)
$$

Similarly to the pure case we choose the approximants only with linear denominators. Treating problems in such a way allows to obtain a stable mixed fixed point, $u^{*}>0, v^{*}>0$, of the model already in the two-loop approximation level, which is demonstrated in figure 1 by plotting the resummed $\beta$-functions of the diluted Ising model. The mixed fixed point is stable thus proving the crossover of the 3DIM to a new critical regime under dilution. The corresponding values of the fixed mixed point coordinates, critical exponents and eigenvalues of the stability matrix are given in the table VI. One can see that increasing the order of approximation the results approach the values both of experiment [5] and theory $[35,36]$. However, on the level of five-loop order the applied resummation techniques do not lead to a real root for the mixed fixed point. It is expected that applications of more sophisticated resummation procedures incorporating the higher order behaviour, still unavailable, will permit to improve the estimates of the critical exponents in the 2-4 loop level as well as to obtain them in the fifth order.

One can state that while in the case of the pure Ising model, where the $\varepsilon$-expansion and the $3 d$-theory analysis of the RG-functions are of equal usefulness, the interpretation of these in the diluted model case can be done only in the framework of the $3 d$-theory analysis. The application of the $\sqrt{\varepsilon}$-expansion remains to be valid only near the upper critical dimension $d=4$. This conclusion holds at least within the discussed resummation schemes. 


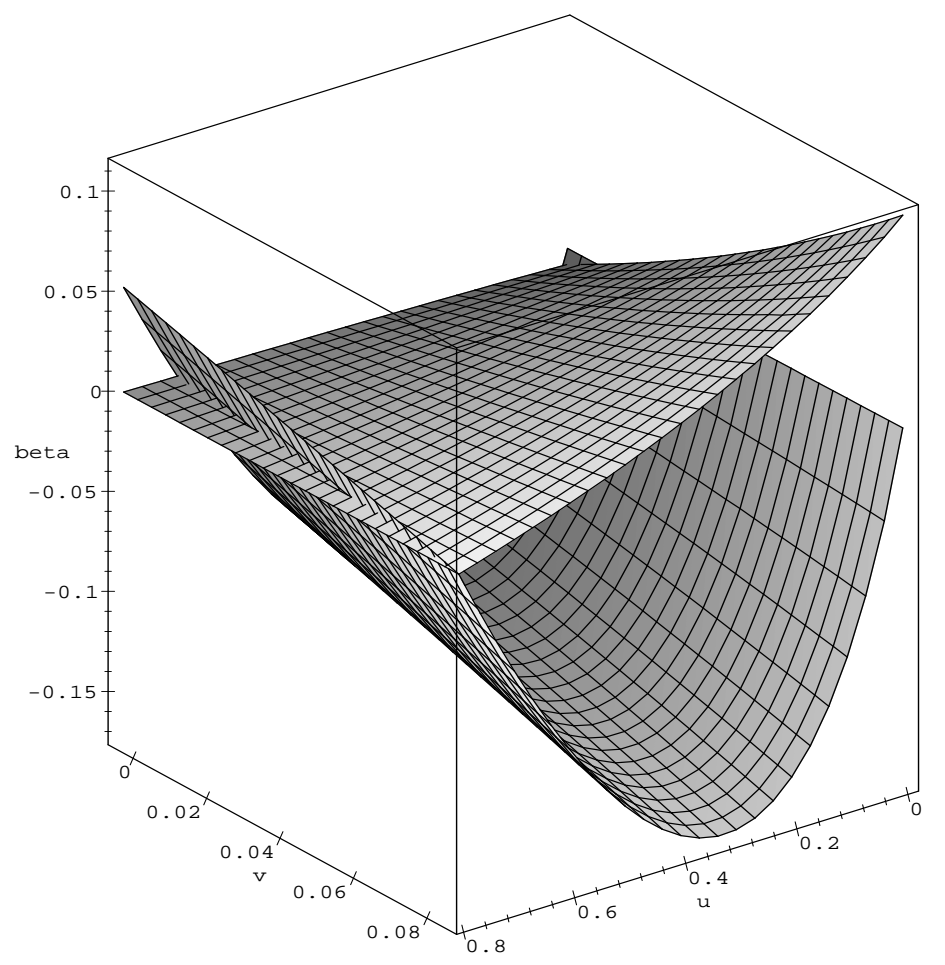

Fig. 1. The Chisholm-Borel resummed $\beta$-functions of the 3DIM in the two-loop approximation. The plain surface corresponds to the $\beta_{v}$-function. Note the presence of the mixed fixed point $u^{*}>0, v^{*}>0$. The intersection of $u$ and $v$ plains is chosen to show the stable fixed point $u^{*}=0.7962, v^{*}=0.0862$ corresponding to a new critical behaviour.

\section{CONCLUSIONS}

We conclude from the above analysis that whereas for the pure Ising model both $\varepsilon$-expansion and $3 d$-technique give reliable results for the numerical values of the critical exponents, in the case of the diluted Ising model the bad convergence properties of the $\sqrt{\varepsilon}$-expansion do not allow to obtain reliable values at $d=3$. The direct resummation of the $\mathrm{RG}$-functions at $d=3$ in the minimal subtraction scheme allows to obtain estimates for the critical exponents without involving information of the large order expansion term behaviour. However one encounters difficulties in the five-loop level, since some of the roots of the $\beta$-function (just those of the relevant mixed fixed point) become complex. We suppose that the fixed $d$ approach, both within the massive [33-38] and minimal subtraction [39] schemes, remains the only reliable way to study critical behaviour of the model by means of RG technique.

One may use the results of the resummation for the calculation of effective critical exponents [48], relevant for the behaviour in the non asymptotic regime. Such a calculation is of special interest for the diluted Ising model since it has been observed that the critical exponents of this model are concentrationally dependent $[9,10,49,50]$. This would contradict the universality hypothesis. However, the concentration dependence of the critical exponents may be explained by noting that the system still is not in the asymptotic regime and its behaviour is governed by effective critical exponents. The results for these exponents will be published elsewhere.
[1] R. Brout, Phys. Rev. 115, 824 (1959).

[2] A. B. Harris, J. Phys. C 7, 1671 (1974).

[3] R. J. Birgeneau, R. A. Cowley, G. Shirane, H. Joshizawa, Phys. Rev. B 27, 6747 (1983).

[4] P. W. Mitchell, R. A. Cowley, H. Yoshizawa, P. Böni, Y. J. Uemura, R. J. Birgeneau, Phys. Rev. B 34, 4719
(1986)

[5] T. R. Thurston, C. J. Peters, R. J. Birgeneau, Phys. Rev. B 37, 9559 (1988).

[6] D. Chowdhury, D. Staufer, J. Stat. Phys. 44, 203 (1986).

[7] J. Marro, A. Labarta, J. Tejada, Phys. Rev. B 34, 347 (1986). 
[8] J.-S. Wang, M. Wöhlert, H. Mühlenbein, D. Chowdhury, Physica A 166, 173 (1990).

[9] H.-O. Heuer, Phys. Rev. B 42, 6476 (1990).

[10] H.-O. Heuer, J. Phys. A 26, L333 (1993).

[11] T. Holey, M. Fähnle, Phys. Rev. B 41, 11709 (1990).

[12] T. C. Lubensky, Phys. Rev. B 11, 3573 (1975).

[13] D. E. Khmelnitskii, Zh. Eksp. Teor. Fiz. 68, 1960 (1975).

[14] G. Grinstein, A. Luther, Phys. Rev. B 13, 1329 (1976).

[15] B. Shalaev, Zh. Eksp. Teor. Fiz. 73, 3201 (1977).

[16] C. Jayaprakash, H. J. Katz, Phys. Rev. B 16, 3987 (1977).

[17] E. Brézin, J. C. Le Guillou, J. Zinn-Justin, Phys. Lett. A 44, 227 (1973).

[18] A. A. Vladimirov, D. I. Kazakov, O. V. Tarasov, Zh. Eksp. Teor. Fiz. 77, 1035 (1979).

[19] K. G. Chetyrkin, A. L. Kataev, F. V. Tkachov, Phys. Lett. B 99, 147 (1981); Phys. Lett. B 101, 457 (1981) (Errata).

[20] K. G. Chetyrkin, S. G. Gorishny, S. A. Larin, F. V. Tkachov, Phys. Lett. B 132, 351 (1983).

[21] D. I. Kazakov, Phys. Lett. B 133, 406 (1983).

[22] S. G. Gorishny, S. A. Larin, F. V. Tkachov, Phys. Lett. A $101,120(1984)$.

[23] H. Kleinert, J. Neu, V. Schulte-Frohlinde, K. G. Chetyrkin, S. A. Larin, Phys. Lett. B 272, 39 (1991).

[24] L. Lipatov, Sov. Phys. JETP 45, 216 (1977).

[25] E. Brézin, J. Le Guillou, J. Zinn-Justin, Phys. Rev. D 15, 1544 (1977).

[26] E. Brézin, G. Parisi, J. Stat. Phys. 19, 269 (1978).

[27] J.-P. Eckmann, J. Magnen, R. Seneor, Commun. Math. Phys. 39, 251 (1975).

[28] G. A. Baker, B. G. Nickel, M. S. Green, D. I. Meiron, Phys. Rev. Lett. 36, 1351 (1976).

[29] G. A. Baker, B. G. Nickel, D. I. Meiron, Phys. Rev. B 17, 1365 (1978).

[30] D. I. Kazakov, O. V. Tarasov, D. V. Shirkov, Teor. Mat. Fiz. 38, 15 (1979).

[31] J. C. Le Guillou, J. Zinn-Justin, Phys. Rev. Lett. 21, 3976 (1980).
[32] J. C. Le Guillou, J. Zinn-Justin, Phys. Rev. Lett. 39, 95 (1977).

[33] G. Jug, Phys. Rev. B 27, 609 (1983).

[34] I. O. Mayer, A. I. Sokolov, Fiz. Tverd. Tela (Leningrad) 26, 3454 (1984)

[35] I. O. Mayer, A. I. Sokolov, B. N. Shalaev, Ferroelectrics 95, 93 (1989)

[36] I. O. Mayer, J. Phys. A 22, 2815 (1989).

[37] Yu. Holovatch, M. Shpot, J. Stat. Phys. 66, 867 (1992).

[38] Yu. Holovatch, T. Yavors'kii, Cond. Matt. Phys. (Lviv) iss. 11, 87 (1997); Yu. Holovatch, T. Yavors'kii, J. Stat. Phys., 1998 (submitted).

[39] H. K. Janssen, K. Oerding, E. Sengespeick, J. Phys. A 28, 6073 (1995).

[40] H. Kleinert, V. Schulte-Frohlinde, Phys. Lett. B 342, 284 (1995).

[41] B. N. Shalaev, S. A. Antonenko, A. I. Sokolov, Phys. Lett. A 230, 105 (1997).

[42] R. Schloms, V. Dohm, Europhys. Lett. 3, 413 (1987).

[43] R. Schloms, V. Dohm, Nucl. Phys. B 328, 639 (1989).

[44] We consider the case $p=0$ in the present calculations. More detailed analysis of the parameter $p$ choice will be discussed elsewhere.

[45] Such a choice is motivated by general suggestions about sign-alternating of the RG-functions expansions in couplings; more detailed analysis which involves application of general approximants and needs enhanced computings will be published elsewhere.

[46] One expects improving the results if more perfect resummation techniques using large order behaviour information are applied.

[47] The resummation was performed introducing into the $\sqrt{\varepsilon}$-expansion an auxiliary parameter $\tau$ by a substitution $\epsilon \rightarrow \epsilon \tau^{2}$, putting $\tau=1$ in the final results.

[48] E. K. Riedel, F. J. Wegner, Phys. Rev. B 9, 294 (1974).

[49] V. V. Prudnikov, A. N. Vakilov, Zh. Eksp. Teor. Fiz. 103, 962 (1993).

[50] H.-O. Heuer, J. Phys. A 26, L341 (1993). 